\title{
ANÁLISIS COMPARATIVO DE LOS MODELOS DINÁMICOS DE UNA TURBINA EÓLICA DE VELOCIDAD FIJA
}

\section{COMPARATIVE ANALISYS OF DINAMICS MODELS OF A FIXED SPEED WIND TURBINE}

\author{
Jesús Guamán-Molina ${ }^{1, *}$, Carlos Vargas-Guevara ${ }^{1}$, Katherin Rodríguez-Mora ${ }^{1}$, \\ Mario García-Carrillo ${ }^{2}$ y Alberto Ríos-Villacorta ${ }^{1}$
}

\section{Resumen}

En este artículo, se presenta un análisis comparativo del comportamiento dinámico de los modelos reducido y completo de una turbina eólica de velocidad fija. La herramienta informática utilizada en la realización de simulaciones es la plataforma Simulink/Matlab. El principal objetivo de este estudio es demostrar la influencia del flujo del estator y del acoplamiento mecánico en la respuesta transitoria de las turbinas eólicas de velocidad fija. Asimismo, se ha analizado la influencia en el comportamiento dinámico de otros parámetros característicos de la turbina eólica, como la potencia reactiva aportada por la batería de condensadores y la inercia del generador y de las palas del rotor eólico.

Palabras clave: Modelos dinámicos, turbina, eólica, respuesta transitoria.

\begin{abstract}
In this article, a comparative analysis of the dynamic behavior of reduced and full of a wind turbine fixed speed models are presented. The tool used in performing simulations is the Simulink/Matlab platform. The main objective of this study is to demonstrate the influence of the stator flux and the mechanical coupling of fixed speed wind turbine dynamic response. It has also analyzed the influence on the dynamic behavior of other characteristic parameters of the wind turbine, such as reactive power supplied by the capacitor bank and the inertia of the generator and wind rotor blades.
\end{abstract}

Keywords: Dynamic Models, Wind Turbine, Transient Response.

\footnotetext{
${ }^{1, *}$ Unidad Operativa de Investigación y Desarrollo de la Facultad de Ingeniería en Sistemas Electrónica e Industrial, Universidad Técnica de Ambato, Ecuador. Autor para correspondencia : jguaman0585@uta.edu.ec

${ }^{2}$ Facultad de Ingeniería en Sistemas Electrónica e Industrial, Universidad Técnica de Ambato, Ecuador.
} 


\section{Introducción}

La integración de energía eólica en un sistema eléctrico exige estudios de integración entre los parques eólicos y el sistema eléctrico. El principal objetivo de los estudios de integración eólica es analizar el comportamiento del sistema eléctrico tanto en régimen permanente como en régimen dinámico. La operación del sistema eléctrico debe garantizar que el funcionamiento del sistema se realice de forma segura, económica y fiable. Por esta razón, es necesario evaluar la influencia de la conexión de un gran número de parques eólicos en la operación del sistema eléctrico, como por ejemplo en estudios de calidad de la energía, de estabilidad estática con ayuda de flujo de cargas, de estabilidad dinámica y transitoria. Evidentemente, el nivel máximo de penetración de energía eólica dependerá directamente de las características del sistema eléctrico analizado: del tipo, capacidad y distribución geográfica de los sistemas de generación convencionales, de los niveles de potencia de cortocircuito, de las capacidades de potencia reactiva, de la capacidad de transmisión de las líneas eléctricas, etc. Un conjunto de procedimientos de operación garantiza la eficiencia y seguridad del funcionamiento de un sistema eléctrico y definen los requisitos técnicos a cumplir por los agentes del sistema eléctrico. En diversos países, con una significativa potencia eólica instalada, se ha introducido una serie de requisitos técnicos específicos a la conexión de parques eólicos, agrupados en procedimientos técnicos de conexión de parques eólicos [1], [2].

Los procedimientos técnicos de conexión establecen una serie de criterios de operación al control de potencia activa y reactiva, definen los perfiles de los huecos de tensión que han de soportar las instalaciones eólicas y proporcionan un conjunto índices de evaluación de la calidad de la energía eólica, como, por ejemplo, tasa de distorsión armónica o niveles de flicker.

En función de las características técnicas, de operación y fiabilidad del sistema eléctrico se especifican los requisitos mínimos que han de cumplir los parques eólicos frente a contingencias severas como los huecos de tensión. Los huecos de tensión son reducciones instantáneas de la tensión ante la ocurrencia de cortocircuitos trifásicos equilibrados o desequilibrados. Los parques eólicos deberán adoptar las medidas necesarias para no desconectarse frente a huecos de tensión. En España, el operador del sistema eléctrico - Red Eléctrica de España, REE - publicó el Procedimiento de Operación 12.3 [3], que establece una curva de tensión-tiempo, específicamente asociada a la magnitud y duración de las tensiones a soportar por una instalación eólica, ante cortocircuitos trifásicos, sin desconectarse de la red eléctrica, zona gris de la Figura 1.

El procedimiento de operación 12.3, además, establece los valores de potencia reactiva generada o consumida en función del nivel de profundidad del hueco de tensión, asociada a una curva de intensidad reactiva admisible-tensión en valores por unidad, en el nodo de conexión, Figura 2. Se considera, por tanto, que los parques eólicos deben generar potencia reactiva en el punto de conexión durante el período de hueco de tensión, zona gris de la Figura 2.

A un nivel de tensión superior a $0.85 \mathrm{pu}$ en el punto de conexión, el parque puede generar o consumir potencia reactiva en función de las características del generador eléctrico empleado. En el presente artículo se simula y analiza la respuesta dinámica de diferentes modelos matemáticos de una turbina eólica equipada con generadores asíncronos de jaula de ardilla.

El principal objetivo del estudio consiste en demostrar la importancia de considerar el flujo magnético del estator y la flexibilidad del acoplamiento mecánico en el análisis transitorio de las turbinas de velocidad fija ante huecos de tensión simétricos.

Los parques eólicos de velocidad fija en régimen permanente consumen potencia reactiva. Así, una vez conocidos los límites de funcionamiento dinámico de los parques eólicos, en casos de huecos de tensión, los estudios de estabilidad transitoria a realizarse se comparan con las curvas de requisitos mínimos, establecidos por el operador del sistema eléctrico. Los resultados obtenidos del estudio comparativo han demostrado que el uso de un modelo matemático del generador de inducción de $5 .^{\circ}$ orden y un modelo de acoplamiento mecánico de dos masas incrementa el margen de estabilidad transitoria de las turbinas eólicas de velocidad fija.

Por tanto, se recomienda este modelo de turbina de velocidad fija para analizar el efecto de diferentes parámetros mecánicos y eléctricos en la respuesta transitoria de la turbina eólica de velocidad fija, así como en los típicos estudios dinámicos de integración de parques eólicos, formados por este tipo de turbinas eólicas.

\section{Generador asíncrono de inducción}

El uso de generadores asíncronos es atractivo debido a la robustez o bajo coste de estos convertidores electromecánicos. Las turbinas eólicas de velocidad fija están equipadas con generadores de inducción de jaula de ardilla, conectados directamente a la red eléctrica. El acoplamiento mecánico existente entre las palas del rotor de la turbina eólica y el generador eléctrico a través de una multiplicadora, puede representarse con ayuda de un modelo de dos masas o de una masa. En el caso del modelo de dos masas, el rotor eólico, que engloba a las palas, el buje y al eje de baja velocidad, y el eje de alta velocidad del generador eléctrico se representan como dos masas separadas, conectadas entre sí por un acoplamiento flexible. La caja multiplicadora establece un acoplamiento flexible entre los dos ejes de la turbina que giran a diferentes velocidades. 


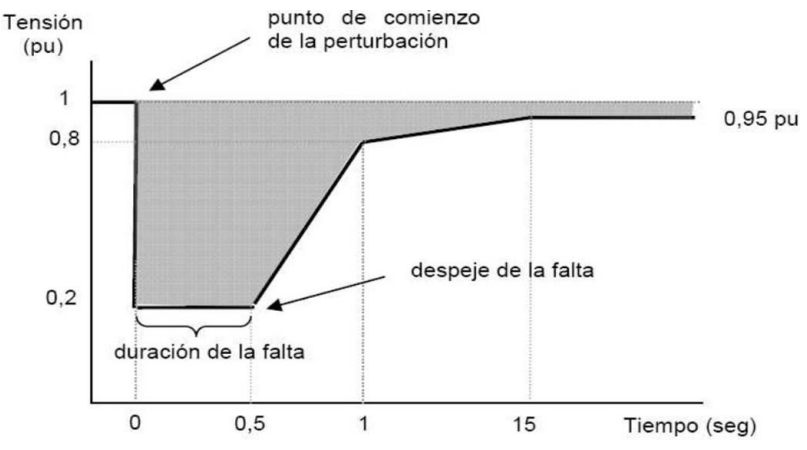

Figura 1. Curva de tensión-tiempo admisible en el punto de conexión [3].

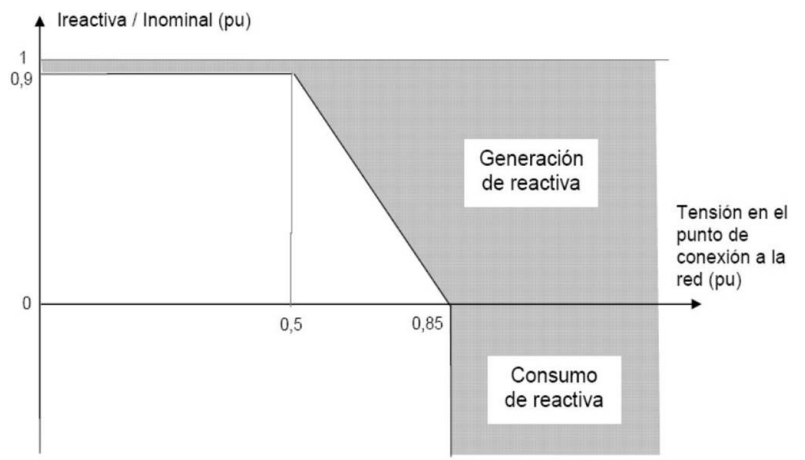

Figura 2. Curva de intensidad reactiva admisible - tensión [3].

En el modelo de una masa, el rotor eólico, la multiplicadora y el eje de alta velocidad del generador eléctrico se representan por una única masa puntual, despreciando la elasticidad existente entre el eje de alta y el eje de baja velocidad. Además, debido al rígido acoplamiento entre el sistema mecánico y el sistema eléctrico de la turbina, las fluctuaciones del par mecánico de la turbina eólica son transmitidas desde el eje de baja velocidad al eje de alta velocidad del generador eléctrico y consecuentemente, aparecen como fluctuaciones de la potencia eléctrica generada.

La potencia reactiva absorbida por el generador de inducción depende de la carga de la turbina y se incrementa con el aumento de la potencia eléctrica generada, proporcional a la velocidad del viento. El factor de potencia de los generadores de inducción es de alrededor de $0.85-0.90$ inductivo. Este factor de potencia exige la instalación de baterías de condensadores que compensen el consumo de potencia reactiva. El generador de inducción posee una característica de par-deslizamiento estrechamente relacionada con la tensión en bornes de la máquina y que afecta al comportamiento dinámico de la turbina de velocidad fija. Durante una contingencia severa, un hueco de tensión profundo provoca un incremento del deslizamiento del generador de inducción. La intensidad del estator y, por tanto, el consumo de potencia reactiva también se incrementan. Adicionalmente, las baterías de condensadores generan menos potencia reactiva, puesto que dependen del cuadrado de la tensión, por lo que el efecto del hueco de tensión se ve intensificado. Si el hueco de tensión decrece hasta el punto que el par amortiguador del generador de inducción es inferior al par mecánico proporcionado por el viento, el rotor del generador se acelerará, desconectando la turbina. Para evitar la aparición de un colapso de tensión es necesario instalar sistemas dinámicos de compensación de potencia reactiva como los SVC o STATCOM [4], [5], [6].

Diferentes estudios evalúan el comportamiento dinámico de las turbinas eólicas de velocidad fija en términos del tiempo crítico de despeje de falta, [7], [8], [9], [10], definido como el máximo tiempo de duración de una falta sin que la turbina se desconecte. La influencia de diversos parámetros eléctricos y mecánicos sobre el tiempo crítico de despeje de falta no consideran los transitorios en el flujo del estator.

En otros trabajos, la respuesta de estabilidad dinámica es evaluada en términos de la recuperación de la tensión, sin embargo, el modelo matemático del acoplamiento mecánico es representado con ayuda del modelo de una masa, ignorando la flexibilidad aportada por la multiplicadora al acoplamiento mecánico entre las palas del rotor eólico y el eje del generador eléctrico [4], [11], [12]. Estas excesivas simplificaciones pueden conducir a resultados y conclusiones erróneas. Puesto que los operadores de sistemas eléctricos han establecido requisitos de incremento de la capacidad para soportar huecos de tensión en las instalaciones eólicas, en función de un tiempo máximo de duración del hueco, parece más lógico analizar el comportamiento dinámico de los generadores de inducción en términos de tensión y velocidad de giro del generador eléctrico en lugar del tiempo crítico de despeje de falta.

\section{Esquema de análisis comparativo}

En la Figura, se muestra el diagrama representativo del esquema eléctrico utilizado en el estudio comparativo y una representación esquemática de los cuatro modelos matemáticos propuestos para la turbina eólica, empleados en el estudio comparativo dinámico frente a huecos de tensión. Se han incluido dos modelos matemáticos del generador de inducción de jaula de ardilla: el modelo completo y el reducido. El primero es el también conocido como modelo de $5 .^{\circ}$ orden que incluye los transitorios del flujo del estator. Por el contrario, el modelo reducido o de $3{ }^{\text {er }}$ orden es aquel en el que el comportamiento dinámico del estator se desprecia. El acoplamiento mecánico entre las palas y el generador eléctrico ha sido representado por sus dos modelos matemáticos más utilizados en la literatura especializada: el modelo flexible o de dos masas y el modelo inflexible o de una sola masa. 


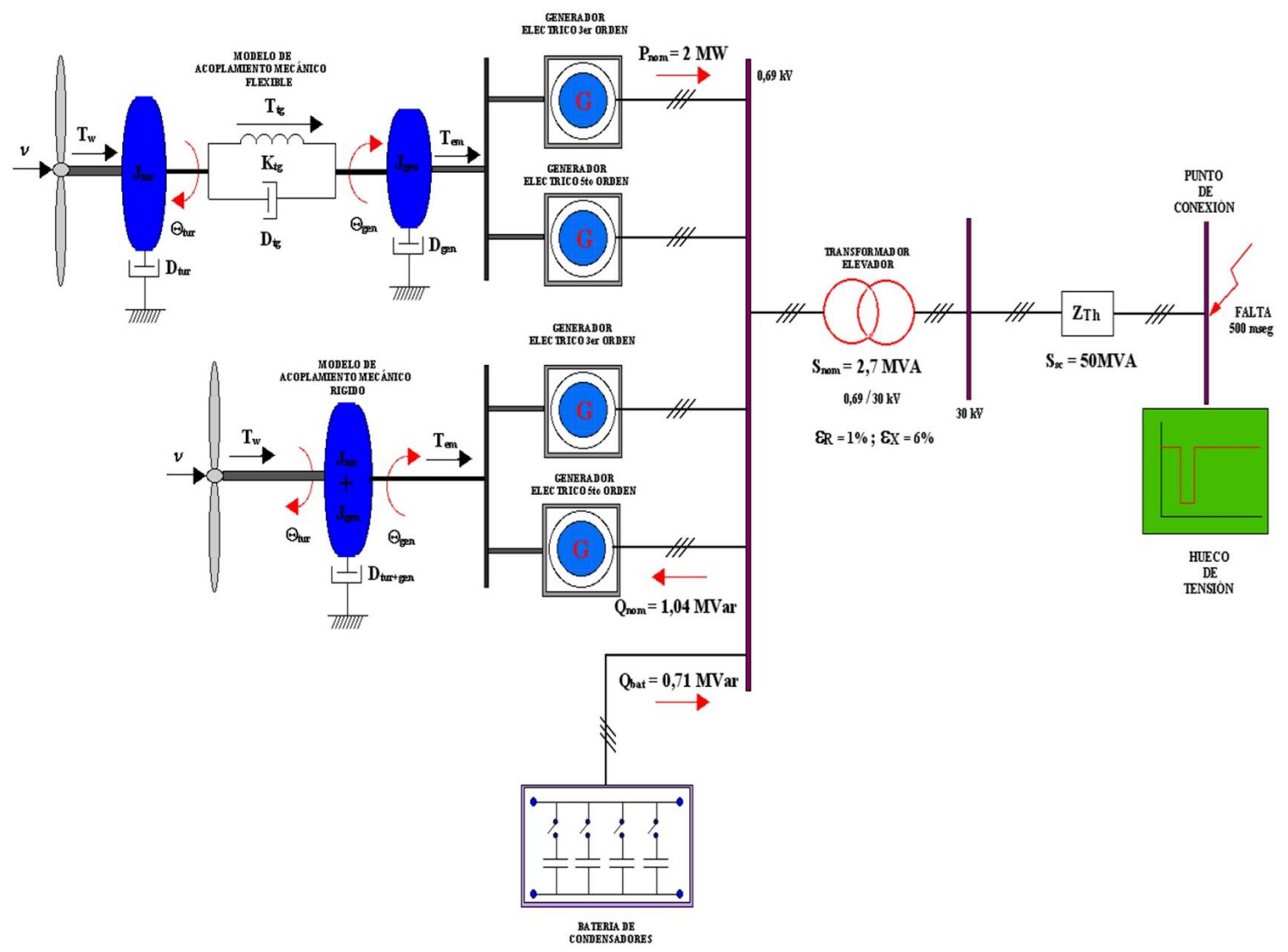

Figura 3. Esquema eléctrico utilizado en el análisis comparativo de los diferentes modelos matemáticos.

Los modelos matemáticos del acoplamiento mecánico y del generador de inducción de la turbina eólica de velocidad fija han sido implementados en el programa Simulink/Matlab.

Se ha incluido, además, el sistema de compensación de potencia reactiva, basado en baterías de condensadores, el transformador elevador de baja a media tensión y la impedancia del equivalente Thevenin hasta el punto de conexión a la red eléctrica.

La turbina eólica está compuesta por un generador de inducción de jaula de ardilla de una potencia nominal de 2 MW y tensión nominal de $690 \mathrm{~V}$. El generador asíncrono es conectado a la red de media tensión de $20 \mathrm{kV}$ a través de un transformador elevador de 2.7 MVA y una relación de transformación de $0.69 / 20 \mathrm{kV}$.

Los parámetros mecánicos de la turbina eólica, y eléctricos del generador asíncrono son dados en la Tabla 1 y 2 respectivamente.

En la Figura 4, se muestra la implementación práctica del estudio comparativo de los diferentes modelos matemáticos de una turbina eólica de velocidad fija en la plataforma de simulación Matlab/Simulink.

\section{Criterios de análisis comparativo}

Para investigar el efecto de los transitorios en el estator y del modelo de acoplamiento mecánico en el comportamiento dinámico de una turbina eólica de velocidad fija se ha realizado un estudio comparativo de los diferentes modelos matemáticos, descritos en el apartado anterior.

En este estudio, se usa la respuesta de tensión después de una falta simétrica trifásica como un criterio de estabilidad dinámica. Asimismo, se determina el tiempo crítico de despeje de falta como criterio complementario de estabilidad dinámica.

Además, se analiza la respuesta de intensidad en el estator, la velocidad de giro del generador y del rotor eólico, la potencia activa y reactiva del generador eléctrico.

En todos los casos analizados, el tiempo de duración de la falta es de $500 \mathrm{~ms}$ y la caída de tensión alcanza el $20 \%$ de la tensión nominal en el punto de conexión, de acuerdo con lo establecido en el procedimiento de operación 12.3. Generalmente, el tiempo típico de despeje de una falta trifásica está en el orden de los 100 - 200 ms. Cuando se investigan oscilaciones 
Tabla 1. Parámetros de la turbina eólica.

\begin{tabular}{lc}
\hline Parámetros de la turbina eólica & Unidad de medida \\
\hline Velocidad de arranque, $v_{\text {cut-in }}$ & $4 \mathrm{~m} / \mathrm{s}$ \\
Velocidad nominal del viento, $v_{n o m}$ & $15 \mathrm{~m} / \mathrm{s}$ \\
Velocidad de giro nominal (turbina eólica), $\omega_{\mathbf{r}_{n o m}}$ & $17 \mathrm{rpm}$ \\
Velocidad de giro nominal (generador), $\omega_{\mathbf{g}_{\text {nom }}}$ & $1510,5 \mathrm{rpm}$ \\
Ángulo de calado, $\beta$ & $0^{\circ}$ \\
Diámetro del rotor, $D_{\text {pala }}$ & $76 \mathrm{~m}$ \\
Longitud de la pala, $L_{\text {pala }}$ & $37 \mathrm{~m}$ \\
Área barrida, $A$ & $4500 \mathrm{~m}^{2}$ \\
Inercia de la turbina eólica, $J_{r}$ & $5,35 \times 10^{6} \mathrm{~kg} \mathrm{~m}^{2}$ \\
Constante de inercia de la turbina eólica, $H_{r}$ & $3,75 \mathrm{seg}$ \\
Rigidez del acoplamiento mecánico, $K_{t g}$ & $74,65 \mathrm{pu}$ \\
Amortiguamiento del acoplamiento mecánico, $D_{t g}$ & $5,0 \mathrm{pu}$ \\
Relación de la multiplicadora, $n_{\text {gear }}$ & 93,75 \\
\hline
\end{tabular}

Tabla 2. Parámetros del generador inducción.

\begin{tabular}{lc}
\hline Parámetros del generador de inducción & Unidad de medida \\
\hline Potencia nominal, $P_{\text {nom }}$ & $2 \mathrm{MW}$ \\
Potencia nominal de la batería de condensadores, $Q_{n o m}$ & $918,2 \mathrm{kVar}$ \\
Tensión nominal, $U_{\text {nom }}$ & $690 \mathrm{~V}$ \\
Frecuencia nominal, $f_{\text {nom }}$ & $50 \mathrm{~Hz}$ \\
Deslizamiento nominal, $s_{n o m}$ & $0,7 \%$ \\
Inercia del generador eléctrico, $J_{g}$ & $121,5 \mathrm{~kg} \mathrm{~m}{ }^{2}$ \\
Constante de inercia del generador eléctrico, $H_{g}$ & $0,75 \mathrm{seg}$ \\
Resistencia del rotor, & $0,0015 \Omega$ \\
Resistencia del estator, & $0,0015 \Omega$ \\
Reactancia del rotor, & $0,005 \Omega$ \\
Reactancia del estator, & $0,00714 \Omega$ \\
Reactancia magnetizante, & $1,04742 \Omega$ \\
\hline
\end{tabular}

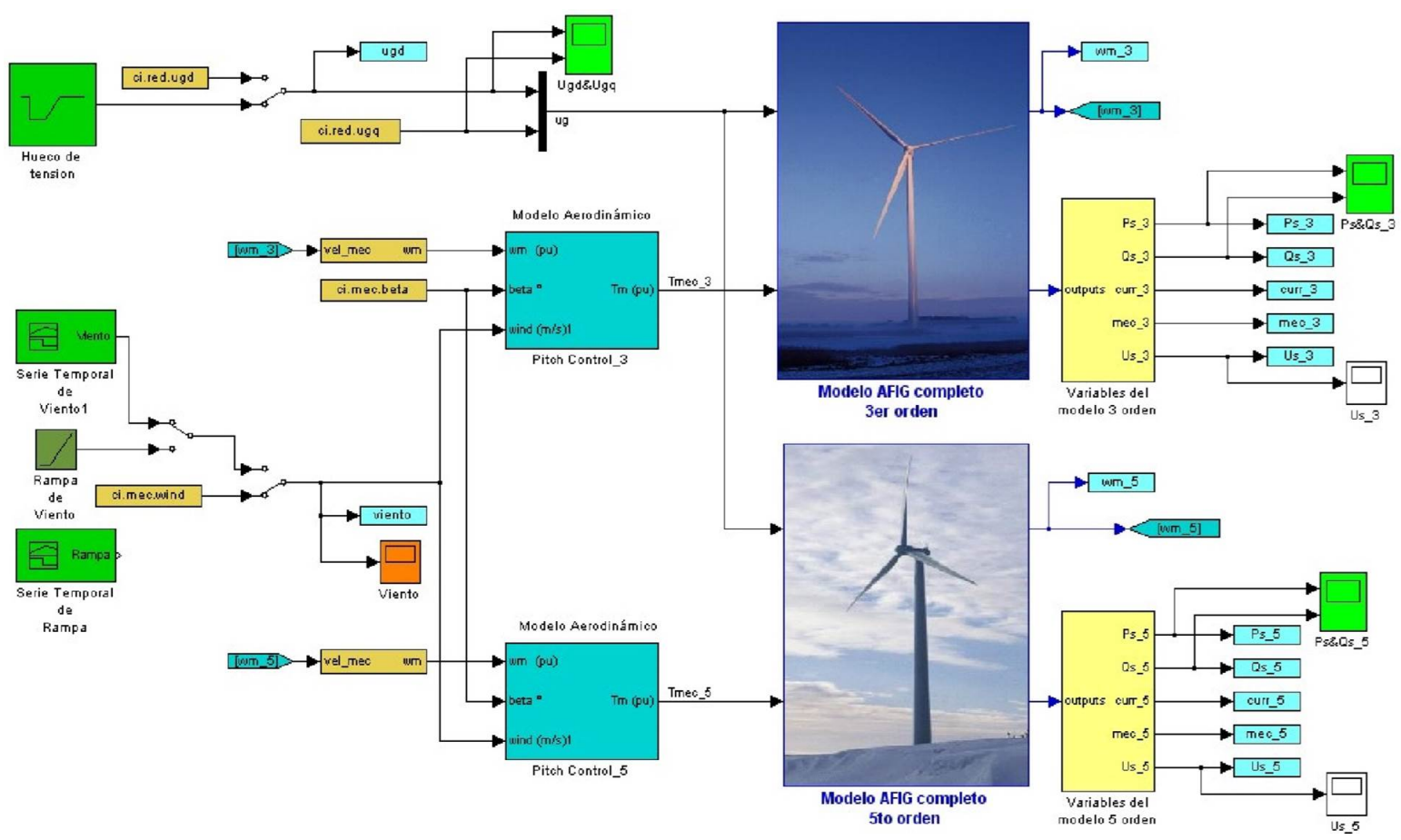

Figura 4. Implementación práctica del análisis comparativo de los modelos matemáticos de una turbina de velocidad fija. 
electromecánicas, el rango de tiempo de interés para estudios dinámicos oscila entre 1 y 10 s.

\section{Análisis de los resultados}

\subsection{Comparación del modelo de generador eléctrico completo y reducido}

En este caso se compara el comportamiento dinámico de un modelo de generador completo frente a un modelo reducido. Ambos modelos utilizan un modelo de acoplamiento flexible. Después de un segundo, se aplica un cortocircuito trifásico en el punto de conexión con la red eléctrica, Figura 5.

La primera falta trifásica se ha despejado después de $500 \mathrm{~ms}$, en tanto que una segunda falta se ha despejado después de $790 \mathrm{~ms}$.

La falta despejada a los 790 ms está relacionada con el tiempo crítico de despeje de falta del modelo reducido del generador eléctrico.

En la Figura 5, se muestra la respuesta de tensión en bornes del generador eléctrico, la velocidad de giro de la turbina eólica y del generador eléctrico, la intensidad en el estator, la potencia activa y reactiva de la turbina eólica. En la Figura 5, cuando se aplica un cortocircuito trifásico en el punto de conexión con la red eléctrica, aparece un hueco de tensión cerca de los terminales del generador de inducción de la turbina eólica. La Figura 5a, muestra como el comportamiento electromecánico del generador se ve afectado.

Las fluctuaciones que aparecen en la tensión, en la intensidad del estator, en la velocidad del rotor del generador eléctrico, en el par electromagnético y en otros parámetros mecánicos y eléctricos se deben a la interacción electromecánica entre la red eléctrica y el acoplamiento mecánico de la turbina eólica. La magnitud y duración de estas oscilaciones depende de las características de torsión del acoplamiento mecánico. Además, el comportamiento dinámico de un generador de inducción se caracteriza por la estrecha relación existente entre los parámetros eléctricos y la velocidad del rotor del generador.

Inicialmente, se describe el impacto de un cortocircuito en el comportamiento eléctrico de un generador de inducción. Si se aplica un cortocircuito muy cerca de los terminales del generador, el flujo del estator decrece instantáneamente. El flujo del rotor, sin embargo, no decrece de forma instantánea debido a la elevada capacidad conductora de la estructura magnética de la jaula de ardilla del rotor, que se opone a cualquier cambio del flujo magnético en cualquier instante de tiempo. Por tanto, en los primeros instantes después de la falta, el generador de inducción inyecta potencia reactiva a la falta contribuyendo a la intensidad de la corriente de falta de cortocircuito con su corriente de desmagnetización, este fenómeno se muestra en la zona ampliada de la Figura 5d. La potencia reactiva aportada por el generador de inducción desmagnetiza el rotor, por lo que el flujo del rotor y la corriente del estator desaparecen sobre los $200 \mathrm{~ms}$, Figura 5f. Cuando se comparan los modelos matemáticos del generador de inducción se observan importantes discrepancias en el comportamiento dinámico de la intensidad de corriente en el estator. El modelo reducido presenta una respuesta de la corriente que no incluye los transitorios de frecuencia fundamental. La magnitud de la corriente prevista por el modelo reducido es, por tanto, menor que el que se obtiene con el modelo completo del generador, esta característica se muestra en la zona ampliada de la Figura 5d. El valor de la magnitud de la corriente del estator es un parámetro muy importante para el diseño de los sistemas de protección de la turbina eólica.

Si se desprecian los transitorios de frecuencia fundamental en el estator, entre los modelos simulados del generador de inducción aparece otra notable discrepancia en los resultados obtenidos para el par de frenado. Evidentemente, esto induce a errores en la respuesta de la velocidad de giro del rotor. Si se utiliza un modelo reducido, la velocidad del rotor se incrementa inmediatamente después de la aparición del cortocircuito, mientras que en un modelo completo, la velocidad del rotor del generador decrece ligeramente en un primer instante y luego se acelera, este fenómeno se aprecia en la zona ampliada de la Figura 5c.

Por otro lado, la velocidad de giro del rotor del generador eléctrico exhibe oscilaciones mecánicas transmitidas por las oscilaciones de par eléctrico del generador, ver la zona ampliada de la Figura 5c. Estas oscilaciones de frecuencia fundamental se deben al flujo estacionario inducido en el entrehierro, durante la desmagnetización del rotor.

Estos transitorios desaparecen en función de la constante de tiempo del estator y son rápidamente amortiguados. La propagación de los transitorios de par desde el eje de alta velocidad al eje de baja velocidad, a través de la multiplicadora, son completamente filtradas por la elasticidad torsional del eje de baja antes de alcanzar el rotor de la turbina, tal como se muestra en la respuesta de la velocidad del rotor de la turbina en la Figura 5b.

Inmediatamente después del cortocircuito, la potencia activa generada se reduce debido a que la tensión del estator alcanza un valor cercano a cero, Figura 5e. La pérdida de potencia eléctrica, inyectada por el generador, causa un desequilibrio entre el par electromagnético de frenado y el par mecánico acelerador (inicialmente invariable), ejercido por el eje de la turbina eólica en función de la velocidad del viento. Por este motivo, el rotor del generador se acelera durante el cortocircuito.

El rotor del generador se acelera por dos razones: una razón es que el rotor acumula energía giratoria 


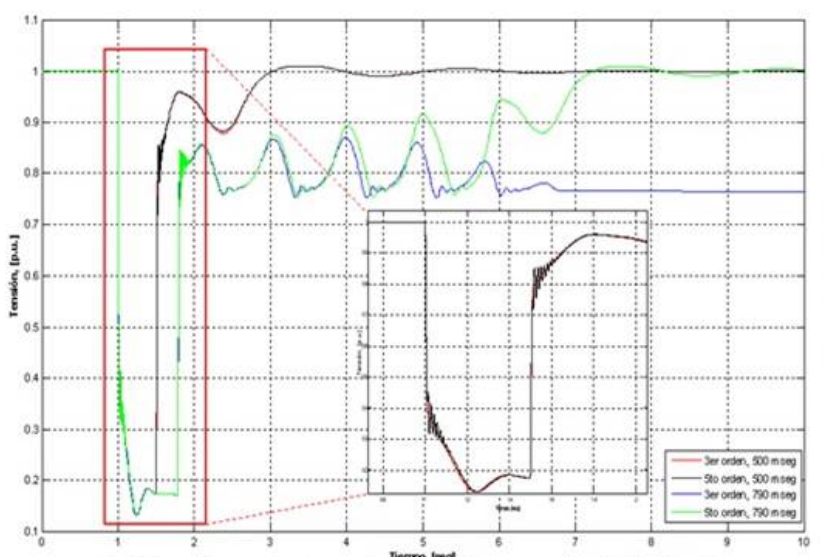

(a) Tensión en los terminales del generador de inducción

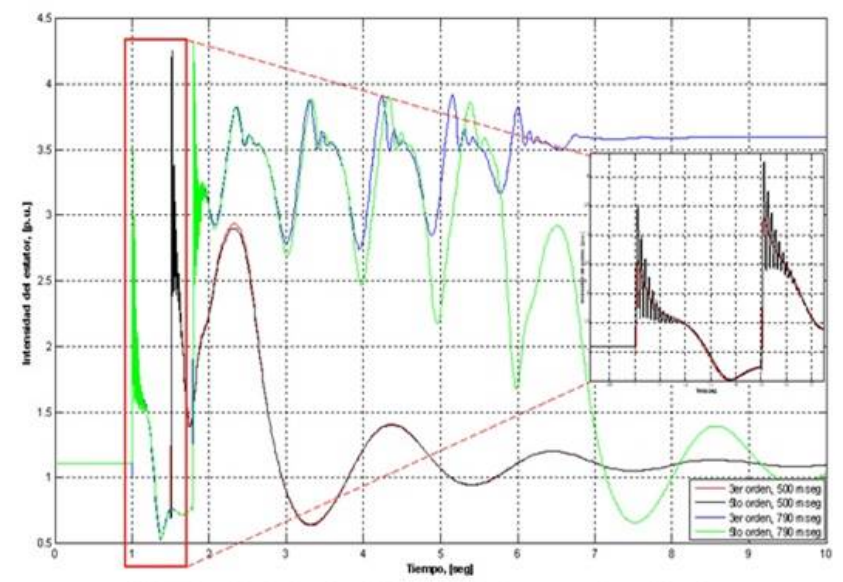

(c) Velocidad de giro del rotor del generador eléctrico

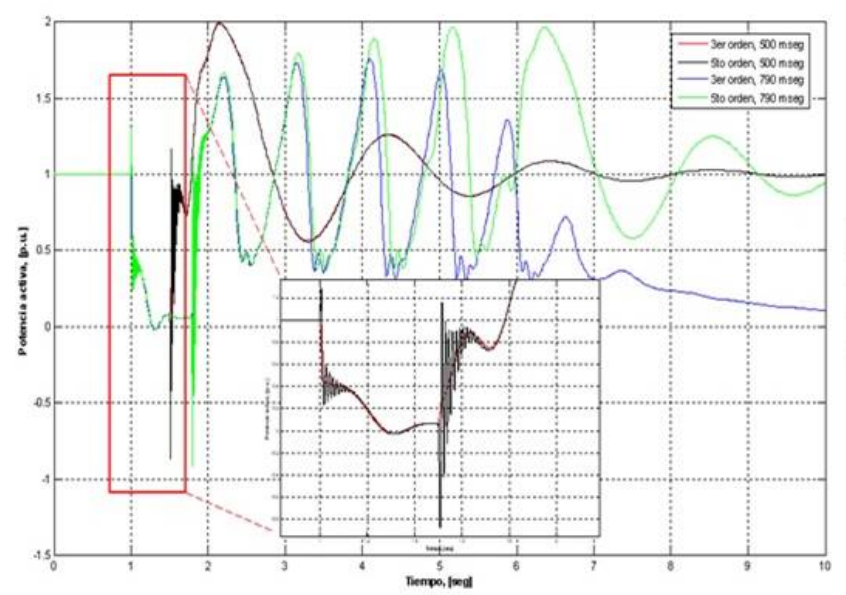

(e) Potencia activa

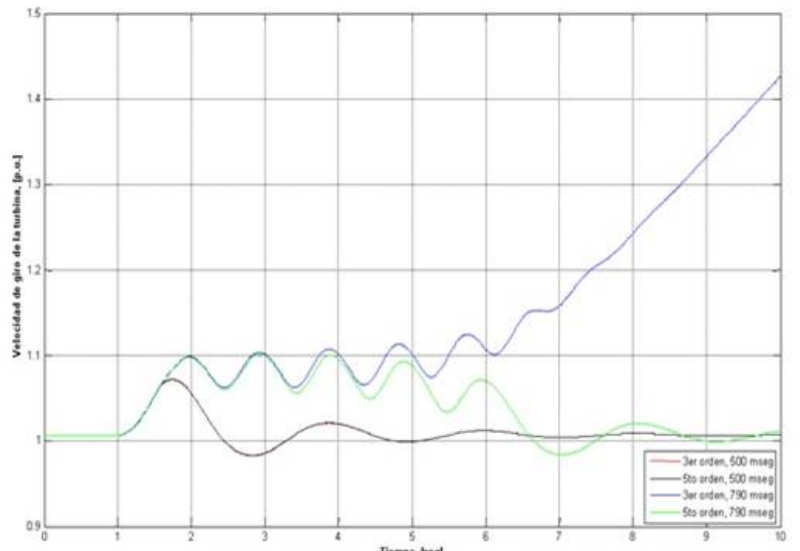

(b) Velocidad de giro del rotor de la turbina eólica

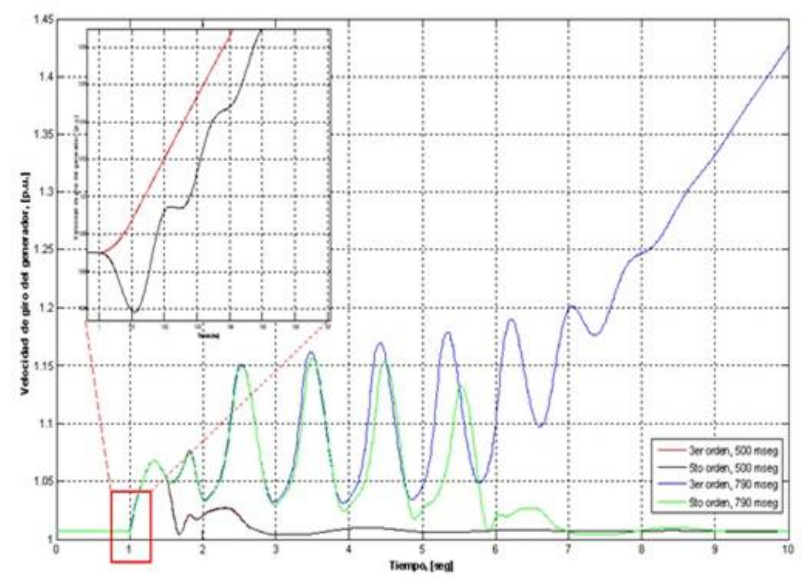

(d) Intensidad del estator

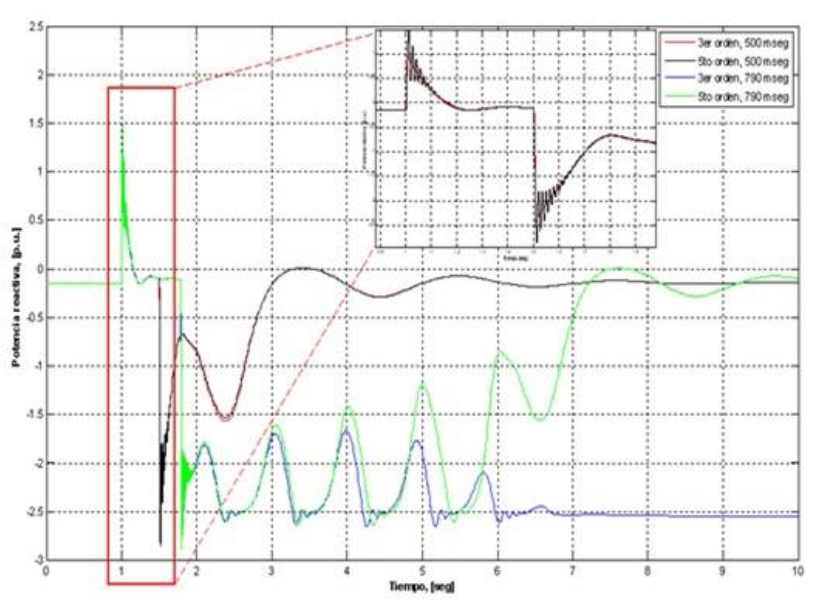

(f) Potencia reactiva

Figura 5. Respuesta transitoria de una turbina eólica de velocidad fija con un modelo flexible de $5 .^{\circ}$ y $3 .^{\text {er }}$ orden ante un cortocircuito trifásico. 
durante la falta, puesto que el viento mantiene una potencia mecánica sobre el eje, aunque no se pueda exportar potencia eléctrica durante la falta. La segunda razón es que el eje de transmisión actúa como un resorte mecánico que se extiende durante la falta. Durante la falta, el par electromagnético se reduce a un valor cercano a cero, lo que extiende el resorte. Debido al diseño aerodinámico de las palas de la turbina eólica no es posible reducir el par mecánico durante el cortocircuito, por lo que el rotor de la turbina eólica también se acelera. Esto da como resultado que el rotor del generador sufra una aceleración adicional.

Cuando se despeja la falta, la tensión recupera su valor inicial, en tanto que el generador asíncrono se ha desmagnetizado y el rotor se ha acelerado, aumentando el deslizamiento negativo de la máquina. En el momento de despeje de la falta, el rotor del generador eléctrico se ha acelerado hasta un valor superior a un determinado valor nominal. Esto implica que el valor de la potencia reactiva demandada por el generador sea significativo. La potencia reactiva consumida por el generador de inducción explica la lenta recuperación de la tensión en bornes de la máquina. Durante la magnetización del rotor, el estator consume un gran valor de intensidad reactiva. Dependiendo de la rigidez de la red eléctrica, esta corriente magnetizante causa una caída de tensión, que reduce la tensión en los terminales del generador por debajo de su valor nominal. Si la duración del cortocircuito es demasiado larga y/o la magnetización del generador de inducción es demasiado lenta, el aumento de la velocidad del rotor será considerable.

El generador eléctrico se puede recuperar satisfactoriamente después de un cortocircuito solo si consigue magnetizarse lo suficientemente rápido para producir un par de frenado, que permita reducir el exceso de velocidad experimentado por el rotor durante la falta. Si el exceso de velocidad es tan grande que el par de frenado no es suficiente para reducir la aceleración del rotor del generador, el consumo de potencia reactiva seguirá siendo apreciable. Precisamente, una técnica para elevar el margen de estabilidad de los generadores asíncronos es reducir el par aerodinámico durante todo el tiempo de duración de cortocircuito. Asimismo, debido a las propiedades elásticas del acoplamiento mecánico, después del despeje de la falta, el tren de transmisión es excitado por oscilaciones torsionales, por lo que la velocidad de giro oscila. Estas oscilaciones son transmitidas a la potencia activa y reactiva del generador eléctrico, Figuras 5e y $5 \mathrm{f}$.

En la Figura 5a, cuando se aplica un cortocircuito de $500 \mathrm{~ms}$, el tiempo de recuperación de la tensión es el mismo para los dos modelos matemáticos comparados del generador asíncrono. Inmediatamente después del cortocircuito, la tensión se reduce hasta un $0.13 \mathrm{pu} \mathrm{y}$ se recupera después de aproximadamente $3 \mathrm{~s}$.
Por otro lado, la duración de las oscilaciones torsionales es de algunos segundos, que corresponde a una frecuencia natural de oscilación del acoplamiento mecánico igual a $1-2 \mathrm{~Hz}$.

Asimismo, en la zona ampliada de la misma figura se muestra el efecto de tomar en cuenta la dinámica del flujo del estator. Estos transitorios son trasladados a la respuesta de tensión durante la falta. Por esta razón, aparecen oscilaciones de frecuencia fundamental inmediatamente después de la aparición del hueco de tensión, inmediatamente después de la aparición de la falta, y durante el proceso de recuperación de tensión. Además, como ya se ha mencionado anteriormente, el comportamiento de la potencia reactiva influye considerablemente en la recuperación de tensión cuando se despeja la falta.

Cuando el rotor del generador se acelera excesivamente, el consumo de potencia reactiva es tan elevado que afecta a la recuperación de la tensión. Este comportamiento de la tensión es observado en la Figura 5a cuando se despeja el cortocircuito a $790 \mathrm{~ms}$, que corresponde al tiempo crítico de despeje de falta del modelo reducido. Por tanto, el modelo reducido presenta una forma de recuperación de la tensión más pesimista que el modelo completo. De la Figura 5c, se observa también, para ambos modelos analizados, violentas oscilaciones de la velocidad del rotor del generador durante el período posterior a la falta.

\subsection{Comparación del modelo de acoplamiento mecánico flexible e inflexible}

En el segundo caso analizado, se compara el comportamiento dinámico del modelo flexible frente al modelo inflexible del acoplamiento mecánico de una turbina eólica. En este caso analizado, se usa el modelo matemático completo del generador asíncrono. $\mathrm{Al}$ igual que en el apartado anterior, se ha aplicado un cortocircuito trifásico en el punto de conexión con la red eléctrica con dos diferentes tiempos de despeje.

La primera falta trifásica se ha despejado después de $500 \mathrm{~ms}$, tal como se establece en el procedimiento de operación 12.3 [3], en tanto que una segunda falta se ha despejado después de $645 \mathrm{~ms}$. La falta despejada a los $645 \mathrm{~ms}$ está relacionada con el tiempo crítico de despeje de falta del modelo inflexible del acoplamiento mecánico.

Los principales parámetros mecánicos y eléctricos del comportamiento dinámico de ambos modelos de la turbina eólica frente a un cortocircuito trifásico se muestran en la Figura 6.

De las Figuras 6a y 6b se observa que la tensión en los terminales del generador y la velocidad de giro del rotor del generador eléctrico para el modelo inflexible recuperan su magnitud anterior a la falta mucho más rápido que en el modelo de dos masas. Se observa también en la Figura 6b que la velocidad del rotor del 


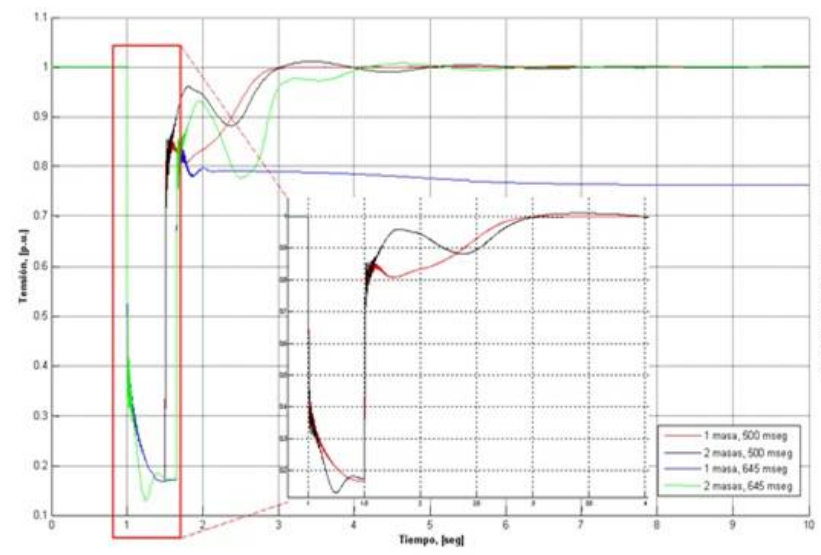

(a) Tensión en los terminales del generador de inducción

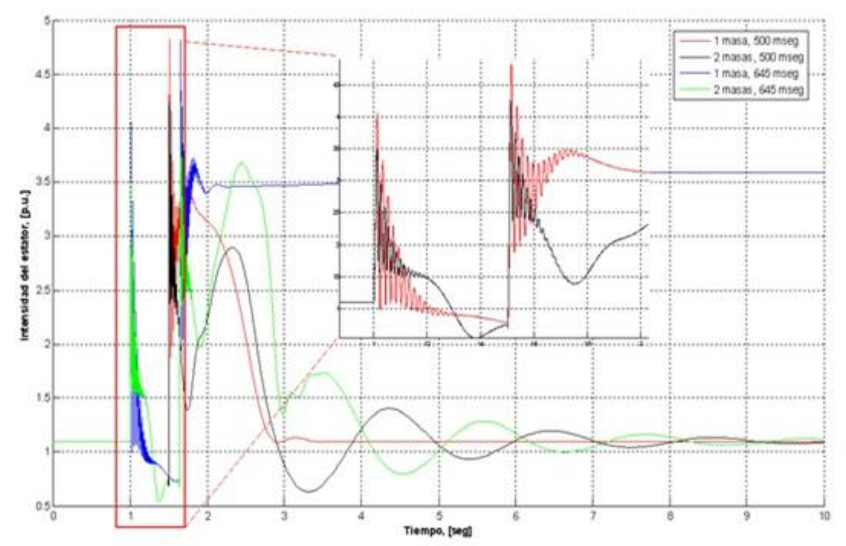

(c) Intensidad en el devanado del estator

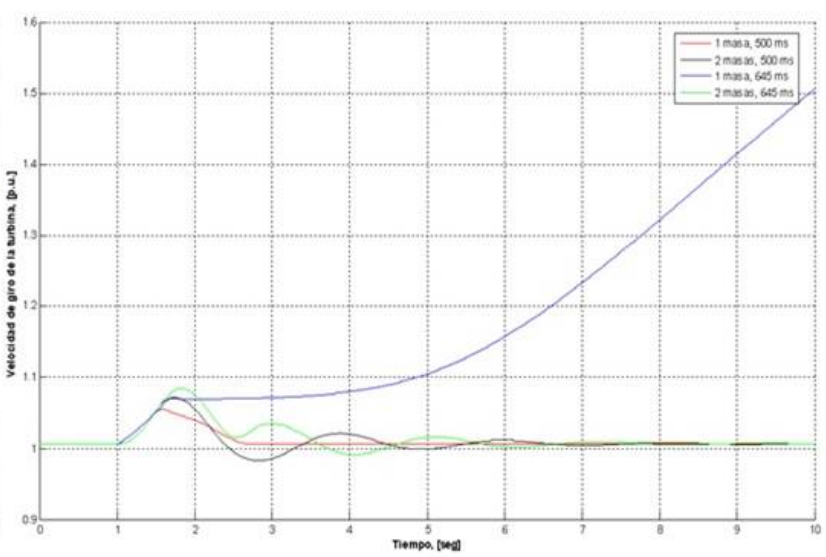

(b) Velocidad de giro

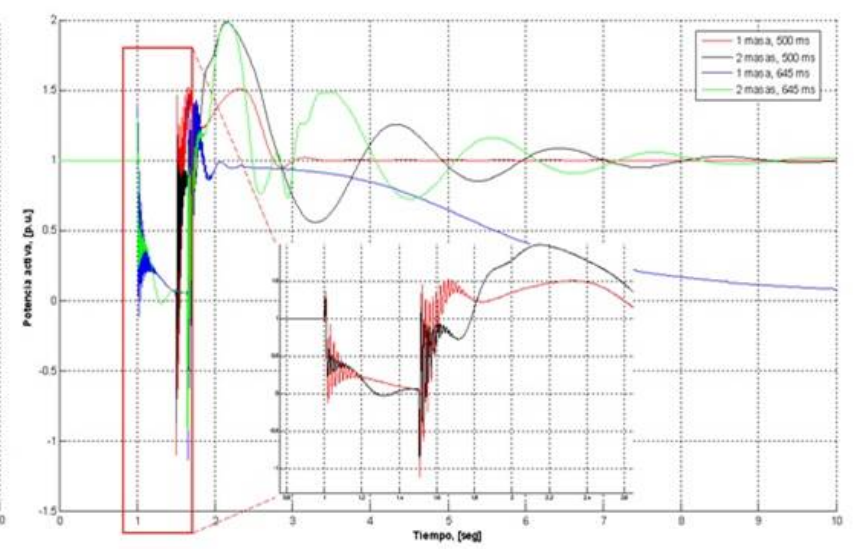

(d) Potencia activa

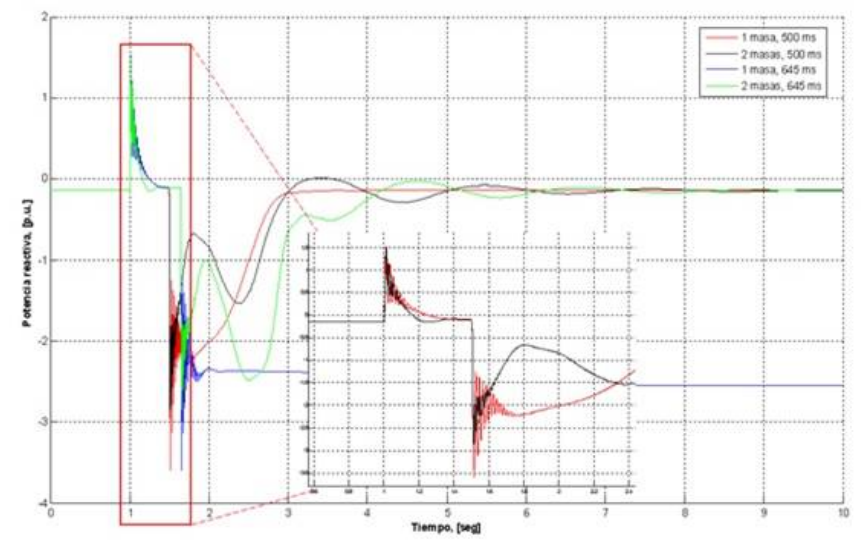

(e) Potencia reactiva

Figura 6. Respuesta transitoria de una turbina eólica con un modelo asíncrono de $5 .^{\circ}$ orden rígido y flexible frente a un cortocircuito trifásico. 
generador para el modelo flexible está sujeto a relativamente elevadas oscilaciones mientras que el del modelo inflexible recupera su valor en régimen permanente sin ninguna oscilación.

Los resultados de las simulaciones relacionadas con la respuesta de tensión y la velocidad del rotor del generador para una duración de la falta de $645 \mathrm{~ms}$ son presentados en las Figuras 6a y Figura 6b.

Como se muestra en la Figura 6a, en el modelo flexible, la tensión en los terminales del generador asíncrono ha alcanzado su valor anterior a la falta, mientras que en el modelo inflexible se observa un colapso de tensión.

Asimismo, se observan violentas oscilaciones durante el período posterior al despeje de la falta. En la Figura $6 \mathrm{~b}$ se muestra que, en el modelo flexible, la velocidad del rotor del generador recupera de forma gradual su valor anterior a la falta. Por el contrario, la velocidad de giro del rotor del generador relacionada con el modelo inflexible continúa aumentando y alcanza violentas oscilaciones, este comportamiento indica que el generador se hace inestable. Estas oscilaciones se trasladan a los parámetros eléctricos del generador de inducción como la potencia eléctrica, la potencia reactiva y la intensidad del estator como se muestra en las Figuras 6c, 6d y 6e, respectivamente.

Analizando los resultados obtenidos es posible deducir que el valor del tiempo crítico de despeje de falta para un modelo inflexible es mayor que el de un modelo flexible. Por otro lado, las curvas obtenidas con el uso de un modelo flexible difieren significativamente de las obtenidas usando un modelo inflexible.

En el caso del modelo inflexible no se observan importantes fluctuaciones ni en la velocidad de giro del rotor ni en el perfil de tensión. En el modelo inflexible, las oscilaciones del eje de acoplamiento mecánico son vistas como una ondulación de muy pequeña magnitud en la respuesta de tensión, en consecuencia, este comportamiento no influye en la estabilidad dinámica de tensión. En otras palabras, cuando se utiliza un modelo flexible, las oscilaciones del sistema de acoplamiento mecánico causan fluctuaciones en la velocidad del rotor del generador, que se ven reflejados en los parámetros eléctricos de la turbina eólica. Las fluctuaciones de tensión y su más lenta recuperación después de una falta en la red son resultado del efecto de los transitorios mecánicos del sistema de transmisión de las turbinas eólicas, equipadas con generadores de inducción.

\section{Conclusiones}

Diversos operadores europeos de sistemas eléctricos exigen que las turbinas eólicas, y por tanto, los parques eólicos puedan soportar huecos de tensión. En este sentido, es necesario realizar estudios dinámicos que permitan evaluar la influencia de diferentes paráme- tros eléctricos y mecánicos en la respuesta transitoria de las turbinas eólicas. Los resultados obtenidos del estudio comparativo recomiendan el empleo de un modelo de $5 .^{\circ}$ orden y un modelo flexible de acoplamiento mecánico de la turbina de velocidad fija en la respuesta transitoria en los estudios de integración de parques eólicos en la red eléctrica convencional.

\section{Referencias}

[1] Specifications for Connecting Wind Farms to the Transmission Network, Transmission System Planning Std. ELT1999-411a, 2000.

[2] German Operator E-On. (2001, December) Erganzendenetzanschlussregelnfurwindenergi eanlagen.

[3] Red Eléctrica de España. (2004) Procedimiento de operación 12.3: Requisitos de respuesta frente a huecos de tensión de las instalaciones de producción de régimen especial. REE. España. [Online]. Available: www.ree.es

[4] A. Ríos, "Continuidad de suministro de parques eólicos ante huecos de tensión," Tesis doctoral, Universidad Carlos III de Madrid, España, 2007.

[5] A. Ríos and M. Gascó, "Transient stability of variable speed wind farms during grid fault," Wind Engineering, vol. 37, no. 2, pp. 199-212, 2013.

[6] A. Ríos and D. Guevara, "Influence of the dvr on the transient response of wind farms against voltage sags," Wind Engineering, vol. 39, no. 3, pp. 335-347, 2015.

[7] P. Ledesma, "Análisis dinámico de sistemas eléctricos con generación eólica," Tesis doctoral, Universidad Carlos III de Madrid, España, 2001.

[8] S. K. Salman and A. L. J. Teo, "Windmill modeling consideration and factors influencing the stability of a grid-connected wind power-based embedded generator," IEEE Transactions on Power Systems, vol. 18, no. 2, pp. 793-802, May 2003.

[9] K. U. T. Senjyu, N. Sueyoshi and T. Funabashi, "Stability analysis of grid connected wind power generating system," in Third International Workshop on Transmission Networks for Offshore Wind Farms, Stockholm, 2002, 2002.

[10] J. G. Slootweg, "Wind power: Modeling and impact on power system dynamics," Ph.D. dissertation, Technical University of Delft, December 2003. 
[11] V. Akhmatov, "Analysis of dynamic behaviour of electric power system with large amount of wind power," Ph.D. dissertation, Technical University of Denmark, Denmark, April 2003.

[12] L. Holdsworth, X. G. Wu, J. B. Ekanayake, and N. Jenkins, "Comparison of fixed speed and doubly-fed induction wind turbines during power system disturbances," IEE Proceedings - Generation, Transmission and Distribution, vol. 150, no. 3, pp. 343-352, May 2003. 